\title{
LINEAR HOMEOMORPHISMS OF SOME CLASSICAL FAMILIES OF UNIVALENT FUNCTIONS
}

\author{
FREDERICK W. HARTMANN
}

\begin{abstract}
The extreme points of the closed convex hull of some classical families of univalent functions analytic on the open unit disk, e.g. the convex, $K$, and starlike, $S t$, have recently been characterized. These characterizations are used to determine an explicit representation for the class of linear homeomorphisms of the extreme points of the closed convex hulls of $K$ and $S t$ and thus of the hulls themselves. With the aid of these representations it is shown that every linear homeomorphism of $K$ or $S t$ is a rotation, i.e. convolution with $\{\exp (\operatorname{in} \theta): n=0,1, \ldots\}$. In the way of a positive result: if $\Phi$ is the convex set of analytic functions with positive real part and $f(0)=1$ and $\mathcal{L}$ is a linear homeomorphism of $\mathcal{P}$, then $\mathcal{L}(S t) \subset S t$, but $\mathcal{L}(K) \nsubseteq K$.
\end{abstract}

Some recent papers have been interested in the study of linear operators on $Q$, the space of functions analytic on $\Delta=\{z:|z|<1\}$, which preserve geometric properties of functions belonging to certain well-known subsets of the family of normalized univalent functions, $S$. (For convolution operators and those of order zero see [6], [7], [8]; for general operators see [5], [9], [10].) The main purpose of this paper is to characterize the set of linear homeomorphisms of the extreme points of the closed convex hulls of the convex and starlike functions and thus all linear homeomorphisms of and between these families. In the second part of this paper we study the effects of linear operators on these families where the linear operator is a homeomorphism of $\mathcal{P}$, the functions of positive real part with $f(0)=1$. We first introduce some terminology and preliminary results.

1. Let $\mathbb{Q}$ denote the set of functions analytic in $\Delta$. Then $\mathbb{Q}$ is a locally convex linear topological space with respect to the topology given by uniform convergence on compact subsets of $\Delta$. A complex sequence $x=\left\{x_{n}\right\}_{n=0}^{\infty}$ is called analytic if the corresponding power series $\sum x_{n} z^{n}$ has radius of convergence $r(x)>0$. For each $0<t \leqslant \infty$, let $\mathbb{Q}_{t}$ be the set of all analytic sequences $x$ for which $r(x) \geqslant t$. Then $\mathbb{Q}_{t}$ is an $F K$ space, [11], and $\mathbb{Q}$ and $\mathbb{Q}_{1}$

Presented to the Society, August 27, 1976; received by the editors June 28, 1976.

AMS (MOS) subject classifications (1970). Primary 30A32, 30A40, 30A76.

Key words and phrases. Continuous linear operator, matrix transformation, functions with positive real part, extreme points, closed convex hull, linear homeomorphism, univalent functions, starlike mappings, convex mappings, Krein-Milman theorem. 
can be identified in the natural way. By a continuous linear operator on $Q$ we mean a mapping $\mathcal{L}$ of $\mathbb{Q}$ to $\mathbb{Q}$ which is both linear and continuous, briefly $\mathcal{L}(a f+g)=a \mathfrak{L}(f)+\mathcal{L}(g)$ and if $f_{n} \rightarrow f$ in the topology of $\mathbb{Q}$ then $\mathcal{L}\left(f_{n}\right)$ $\rightarrow \mathscr{L}(f)$ in $\mathbb{Q}$. Each linear operator on $\mathbb{Q}$ can be represented in the following way as a matrix $\mathcal{L}=\left(a_{n k}\right), n, k=0,1,2, \ldots$ If $f(z)=\sum_{n=0}^{\infty} b_{n} z^{n}$, then $\mathcal{L}(f)=\sum_{n=0}^{\infty} c_{n} z^{n}$ where $c_{n}=\sum_{k=0}^{\infty} a_{n k} b_{k}, n=0,1,2, \ldots$ The following theorem, the basis of whose proof can be found in [4], characterizes linear operators from $\mathbb{Q}_{s}$ to $\mathbb{Q}_{t}$.

THEOREM 1. Each of the following are necessary and sufficient for $\mathcal{L}=\left(a_{n k}\right)$ to map $\mathbb{Q}_{s}$ into $\mathbb{Q}_{t}(0<t, s \leqslant \infty)$ :

(i) for each $\beta \in(0, t), \exists \alpha \in(0, s)$ and $M$ such that $\sum_{n}\left|a_{n k}\right| \beta^{n} \alpha^{-k} \leqslant M$ for every $k$,

(ii) for each $\beta \in(0, t), \exists \alpha \in(0, s)$ and $M$ such that $\left|a_{n k}\right| \beta^{n} \alpha^{-k} \leqslant M$ for all $n$ and $k$.

Note that with $s=t=1$ condition (ii) implies that for each $n, x_{n}$ $=\left\{a_{n k}\right\}_{k=0}^{\infty}$ is an analytic sequence with $r\left(x_{n}\right) \geqslant 1 / \alpha>1$.

Let $Q(0)$ be the subspace of all functions in $\mathbb{Q}$ which vanish at the origin and let $S$ be the subset of univalent functions in $\mathscr{Q}(0)$ with $f^{\prime}(0)=1$. Denote by $K$ and $S t$ the subsets of $S$ for which $f(\Delta)$ is respectively convex and starlike with respect to the origin. Let $\mathscr{F} \mathscr{F}$ denote the closed convex hull of any subset $\mathscr{F}$ of $Q$ and $\mathscr{F} \mathcal{F}$ its extreme points. (See [3, Chapter 5] for basic definitions and results.) In [1] it was shown that

$$
\begin{aligned}
& \mathscr{E H} K=\{f: f(z)=z /(1-x z),|x|=1\}, \\
& \mathscr{E} \mathcal{H} S t=\left\{f: f(z)=z /(1-x z)^{2},|x|=1\right\} .
\end{aligned}
$$

LEMMA 1. Let $M$ be the set of infinite matrices $A(\gamma, \theta)=\left(a_{n k}\right)$ generated by

$$
\left[e^{i \theta} \frac{z-\gamma}{z \bar{\gamma}-1}\right]^{n}=\sum_{k=0}^{\infty} a_{n k} z^{k}, \quad n=0,1,2, \ldots
$$

$\theta$ real, $|\gamma|<1\left(\sum_{k=0}^{\infty} a_{0 k} z^{k} \equiv 1\right)$ then

(i) $M$ is a group under infinite matrix multiplication which is isomorphic to the group of bilinear transformations of the closed unit disk onto itself.

(ii) If $A$ is considered as a sequence-to-sequence transformation then $A$ is oneto-one on $\left\{x:\left\{x_{k}\right\}=O(k)\right\}$.

Proof. (i) The desired isomorphism maps $A$ to its corresponding generating bilinear map of $\bar{\Delta}$, and matrix multiplication corresponds to function composition. Note $A(0,0)=I$ and $A^{-1}(\gamma, \theta)=A\left(\gamma e^{i \theta},-\theta\right)$. Associativity follows from the fact that all the generating functions as power series expanded about the origin have radius of convergence greater than one and hence are absolutely convergent on $\bar{\Delta}$.

(ii) Let $x=\left\{x_{k}\right\}=O(k)$. It suffices to show that 


$$
A\left(\gamma_{1}, \theta_{1}\right)\left[A\left(\gamma_{2}, \theta_{2}\right) x\right]=\left[A\left(\gamma_{1}, \theta_{1}\right) A\left(\gamma_{2}, \theta_{2}\right)\right] x
$$

since $M$ is a group. If $A\left(\gamma_{1}, \theta_{1}\right)=\left(a_{n k}\right)$ and $A\left(\gamma_{2}, \theta_{2}\right)=\left(b_{n k}\right)$ and $y_{n}$ $=\sum_{k} b_{n k} x_{k}$, then

$$
\sum_{n} a_{j n} y_{n}=\sum_{n} a_{j n}\left(\sum_{k} b_{n k} x_{k}\right)=\sum_{k}\left(\sum_{n} a_{j n} b_{n k}\right) x_{k}
$$

where the interchange in summation is permissible since $\sum_{k}\left|a_{n k}\right|\left|x_{k}\right|<\infty, n$ $=0,1,2, \ldots$, and $\sum\left|b_{n k}\right|\left|x_{k}\right|<\infty, n=0,1,2, \ldots$ This again follows since the generating functions for $\left(a_{n k}\right)$ and $\left(b_{n k}\right)$ have radius of convergence greater than one and the image of $\bar{\Delta}$ under one generating function is included in the domain of the other.

THEOREM 2. Let $\mathcal{L}$ be a continuous linear operator on $\mathbb{Q}(0)$; then the following statements are equivalent:

(i) $\mathcal{L}$ is a one-to-one mapping of $\mathcal{H} K$ onto $\mathcal{H} K$.

(ii) $\mathcal{L}$ is a one-to-one mapping of $\mathcal{E} \mathcal{H} K$ onto $\mathcal{E} \mathfrak{H} K$.

(iii) $\mathcal{L}=\left(a_{n k}\right)$ where $A=\left(a_{n k}\right) \in M$ (see Lemma 1$)$.

Proof. (ii) $\Rightarrow$ (iii). Let $\mathcal{L}$ be a continuous linear operator on $\mathcal{Q}(0)$ which is a bijection of $\mathcal{E} \mathcal{H} K$. $\mathcal{L}$ is represented as $\left(a_{n k}\right)$. Moreover, $x=\left\{a_{1 k}\right\}_{k=0}^{\infty}$ is an analytic sequence with $r(x)>1$ (Theorem 1). If $f \in \mathcal{E} \mathcal{H} K$ then

$$
f(z)=z /(1-x z), \quad|x|=1 .
$$

Let $x=e^{i \phi}$, then $\mathcal{E}\left(\left\{e^{i k \phi}\right\}_{k=0}^{\infty}\right)=\left\{e^{i n \theta}\right\}_{n=0}^{\infty}$ for some $\theta$. This implies $\sum_{k=0}^{\infty} a_{1 k} e^{i k \phi}=e^{i \theta}$. Hence $\sum a_{1 k} z^{k}$ represents an analytic function on $|z| \leqslant R$, $R>1$, which maps $\bar{\Delta}$ homeomorphically onto itself, thus

$$
\sum a_{1 k} z^{k}=\left[e^{i \alpha} \frac{z-\gamma}{z \bar{\gamma}-1}\right], \text { for some }|\gamma|<1 \text { and } \alpha \text { real. }
$$

By induction it follows that

$$
\sum_{k} a_{n k} z^{k}=\left[e^{i \alpha} \frac{z-\gamma}{z \bar{\gamma}-1}\right]^{n}, \quad n=0,1,2, \ldots
$$

This follows upon equating coefficients of respective power series.

(iii) $\Rightarrow$ (ii). Suppose $A \in M$. Then $A$ is a linear operator on $\mathcal{E S C K}$ into $\mathcal{G} \mathcal{F C K}$. Lemma 1 implies $A$ is $1-1$ on $\mathscr{E F C K}$. Clearly $A$ is onto, hence a linear bijection of $E S C K$.

(ii) $\Rightarrow$ (i). Assume $\mathcal{L}$ is a linear bijection of $\mathcal{E} \mathcal{H} K$. The representation of (iii) again implies $\mathcal{E}$ is $1-1$ on $\mathcal{H} K$. $A$ is into $\mathcal{H} K$. [Let $y \in \mathcal{H} K$, then $\exists y_{\delta} \rightarrow y$ where $y_{\delta}=\sum_{n} a_{n}(\delta) \varepsilon_{n}(\delta), \sum a_{n}=1, a_{n} \geqslant 0, \varepsilon_{n}(\delta)$ an extreme point of $\mathcal{H} K$ (Krein-Milman theorem [3]), but

$$
A\left(y_{\delta}\right)=\sum_{n} a_{n}(\delta) A\left(\varepsilon_{n}(\delta)\right)=\sum_{n} a_{n}(\delta) \varepsilon_{n}^{\prime}(\delta), \quad \varepsilon_{n}^{\prime}(\delta) \in \mathcal{E} \mathcal{H} K .
$$

Hence $A(y)=\lim _{\delta} A\left(y_{\delta}\right) \in \mathcal{H} K$.] Likewise, $A$ is onto $\mathcal{H} K$. 
(i) $\Rightarrow$ (ii). Let $\mathcal{L}$ be a linear bijection of $\mathcal{I} C K$. Then $\mathcal{E}$ is linear and 1-1 on $\mathscr{E} \mathcal{S C K}$. [EFC $K \subset \mathcal{H C} K$ by the Krein-Milman theorem.] Suppose $f \in \mathcal{E} \mathcal{F C} K$ but $\mathscr{L}(f)$ $\notin \mathcal{E} \mathcal{H} K$. Then $\mathfrak{L}(f)=t g_{1}+(1-t) g_{2}, g_{1} \neq g_{2}, g_{1}, g_{2} \in \mathcal{H} K, 0<t<1$, but

$$
f=t \mathfrak{L}^{-1}\left(g_{1}\right)+(1-t) \mathfrak{L}^{-1}\left(g_{2}\right) \notin \mathscr{E} \mathcal{F} K, \quad \mathfrak{L}^{-1}\left(g_{1}\right) \neq \mathfrak{L}^{-1}\left(g_{2}\right) .
$$

Likewise, $\mathcal{L}$ can be shown to be onto $\mathscr{E} \mathcal{F} K$.

For notational convenience we shall write for $f(z) \in \mathbb{Q}(0)$,

$$
f(z)=\sum_{k=0}^{\infty} b_{k} z^{k+1}=b_{0} z+b_{1} z^{2}+\cdots,
$$

and hence $\mathfrak{L}(f)=\sum_{n=0}^{\infty} c_{n} z^{n+1}$, where $c_{n}=\sum_{k=0}^{\infty} a_{n k} b_{k}$. In the sequel we shall also drop the $\exp (i \theta)$ factor in the generating functions for the class $M$, since this factor merely causes a rotation of $\mathcal{L}(f)$ and for our results does not affect the geometry of the range of $\mathfrak{L}(f)$.

THEOREM 3. Let $f(z)=\sum_{k=0}^{\infty} b_{k} z^{k+1} \in \mathbb{Q}(0)$ and $A=\left(a_{n k}\right) \in M$ then

$$
A(f)=b_{0} z /(1-\gamma z)+\sum_{k=1}^{\infty} b_{k} z^{2} \frac{\left(|\gamma|^{2}-1\right)}{(1-\gamma z)^{2}}\left[\frac{\bar{\gamma}-z}{1-\gamma z}\right]^{k-1} ;
$$

equivalently,

$$
A(f)= \begin{cases}z \frac{b_{0} \bar{\gamma}}{\bar{\gamma}-z}+z^{2} \frac{\left(|\gamma|^{2}-1\right)}{(\bar{\gamma}-z)^{2}} f\left(\frac{\bar{\gamma}-z}{1-\gamma z}\right), & z \neq \bar{\gamma}, \\ \frac{\bar{\gamma}}{\left(1-|\gamma|^{2}\right)}\left[b_{0}-\bar{\gamma} b_{1}\right], & z=\bar{\gamma}\end{cases}
$$

Proof. $A(f)=\sum_{k=0}^{\infty} c_{k} z^{k+1}$ where $c_{k}=\sum_{i=0}^{\infty} a_{k i} b_{i}$, thus

$$
A(f)=\sum_{k=0}^{\infty}\left(\sum_{i=0}^{\infty} a_{k i} b_{i}\right) z^{k+1}=\sum_{i=0}^{\infty} b_{i}\left(\sum_{k=0}^{\infty} a_{k i} z^{k+1}\right), \quad|z|<1 .
$$

To obtain expressions for $\sum_{k=0}^{\infty} a_{k i} z^{k+1}$ consider

$$
\phi_{t}(z)=1 /(1-t[(\gamma-z) /(1-\bar{\gamma} z)]) \text {. }
$$

Then $\phi_{t}(z)$ is analytic for $|t|<1$ and $|z| \leqslant 1$.

$$
\phi_{t}(z)=\sum_{n=0}^{\infty} t^{n}\left[\frac{\gamma-z}{1-\bar{\gamma} z}\right]^{n}=\sum_{n=0}^{\infty} t^{n}\left(\sum_{k=0}^{\infty} a_{n k} z^{k}\right)=\sum_{k=0}^{\infty}\left(\sum_{n=0}^{\infty} a_{n k} t^{n}\right) z^{k}
$$

But

(4) $\phi_{t}(z)=\frac{1-\bar{\gamma} z}{1-\gamma t} \frac{1}{1-[(\bar{\gamma}-t) /(1-\gamma t)] z}=\frac{1-\bar{\gamma} z}{1-\gamma t} \sum_{k=0}^{\infty}\left[\frac{\bar{\gamma}-t}{1-\gamma t}\right]^{k} z^{k}$.

Equating coefficients of $z^{k}$ in (3) and (4) gives 


$$
\begin{aligned}
\sum_{n=0}^{\infty} a_{n 0} t^{n} & =\frac{1}{1-\gamma t}, & k & =0, \\
\sum_{n=0}^{\infty} a_{n k} t^{n} & =t \frac{\left(|\gamma|^{2}-1\right)}{(1-\gamma t)^{2}}\left[\frac{\bar{\gamma}-t}{1-\gamma t}\right]^{k-1}, & k & =1,2, \ldots
\end{aligned}
$$

A short calculation gives (1) and (2).

We next obtain a result for linear homeomorphisms of the starlike functions analogous to Theorems 2 and 3. The method of proof is similar to these results so we state only the results.

THEOREM 4. For $\mathcal{L}$ a continuous linear operator on $\mathbb{Q}(0)$ the following statements are equivalent:

(i) $\mathcal{L}$ is a one-to-one mapping of $\mathcal{H S t}$ onto $\mathcal{H} S \mathrm{St}$.

(ii) $\mathcal{L}$ is a one-to-one mapping of EFSt onto EHSt.

(iii) $\mathcal{E}=\left(a_{n k}\right)$ where the matrix is given by

$$
(n+1)\left[\frac{z-\gamma}{z \bar{\gamma}-1}\right]^{n}=\sum_{k=0}^{\infty} a_{n k}(k+1) z^{k}, \quad|\gamma|<1, n=0,1,2, \ldots
$$

If any of (i)-(iii) hold and $f(z)=\sum_{k=0}^{\infty} b_{k} z^{k+1}$ then

$$
\begin{aligned}
\mathcal{E}(f)= & b_{0} z /(1-\gamma z)^{2}+b_{1} z^{2}\left(|\gamma|^{2}-1\right) /(1-\gamma z)^{3} \\
& +\sum_{k=2}^{\infty} b_{k} z^{2} \frac{\left(1-|\gamma|^{2}\right)\left[\left(k+1+|\gamma|^{2}\right) z-2 \bar{\gamma}\right]}{(k+1)(1-\gamma z)^{4}}\left[\frac{\bar{\gamma}-z}{1-\gamma z}\right]^{k-2}
\end{aligned}
$$

If $\mathcal{E}$ is a linear homeomorphism of a compact subset $\mathscr{F}$ of $\mathscr{Q}$ onto $\mathscr{F}^{\prime}$ then $\mathscr{E F F F}^{\prime}=\left\{f^{\prime}: f^{\prime}=\mathfrak{L}(f), f \in \mathcal{E F} \mathcal{F F}\right\}$. Thus if $\mathcal{L}$ is a linear homeomorphism of $K$ or $S t$, it follows with the aid of the Krein-Milman theorem that $\mathcal{L}$ is a linear homeomorphism of the $\mathscr{E F C} K$ or $\mathscr{E F S}$ t respectively. This implies that $\mathcal{L}$ satisfies the hypotheses of Theorems 3 and 4.

THEOREM 5. For $\mathcal{L}$ a linear operator on $\mathcal{Q}(0), \mathcal{L}$ is a linear homeomorphism of $K$ or $S t$ if and only if $\mathcal{L}(f(z))=e^{-i \theta} f\left(e^{i \theta} z\right)$, for some real $\theta$, i.e. $\mathcal{L}$ is a rotation.

Proof. To show the result for $K$ it suffices to exhibit for each linear homeomorphism $\mathcal{L}$ of $\mathcal{E S C} K$ an $f \in K$ such that $\mathcal{L}(f) \notin K$. Assume in formula (2) that $\gamma$ is real and $0<\gamma<1$. Note, if $\gamma=0$ then $\operatorname{ef}(z)=-f(-z)$ (more generally a rotation). Let $f(z)=\frac{1}{2} \log [(1+z) /(1-z)], \operatorname{Im}(f) \in(-\pi / 4, \pi / 4)$, then $f \in K$. By (2)

$$
\mathcal{E}_{\gamma}(f)(z)=\frac{\gamma z}{\gamma-z}+\frac{z^{2}\left(\gamma^{2}-1\right)}{2(\gamma-z)^{2}} \log \left[\frac{(1+\gamma)(1-z)}{(1-\gamma)(1+z)}\right], \quad|z|=1, z \neq \pm 1 .
$$

Since $k(1-z) /(1+z), k>0$, maps $C=\left\{z: z=e^{i t}, 0<t<\pi\right\}$ onto $u$ $=0, v<0$, investigation of $\mathcal{L}_{\gamma}(f)\left(e^{i t}\right), t$ near $0(t>0)$ and $t$ near $\pi(t<\pi)$ shows that the image of the half circle $C$ by $\mathcal{L}_{\gamma}(f)$ has horizontal asymptotes 
$\pi i(1+\gamma) / 4(1-\gamma)$ and $\pi i(1-\gamma) / 4(1+\gamma)$. Thus $\mathfrak{L}_{\gamma}(f)$ can only be convex for $\gamma=0$. For arbitrary $\gamma,|\gamma|<1$, a rotation of $f(z)$ yields a similar result.

To prove the result for $S t$, consider $f(z)=z+a_{2} z^{2}$. Then $f \in S t$ if and only if $\left|a_{2}\right| \leqslant \frac{1}{2}$, [2]. Let $0 \leqslant \gamma<1$ in formula (5) and $a_{2}=\frac{1}{2}$, then $\mathfrak{L}_{\gamma}(f)(z)$ $=\left(z-a z^{2}\right) /(1-\gamma z)^{3}, a=\gamma+\frac{1}{2}\left(1-\gamma^{2}\right)$.

$$
\begin{aligned}
\left.\operatorname{Re}\left\{z \frac{\mathcal{L}_{\gamma}^{\prime}(f)(z)}{\mathcal{L}_{\gamma}(f)(z)}\right\}\right|_{z=1} & =\left.\operatorname{Re}\left\{\frac{1+\gamma z}{1-\gamma z}+\frac{z(\gamma-a)}{(1-\gamma z)(1-a z)}\right\}\right|_{z=1} \\
& =-r(1+\gamma) /(1-r)^{2}<0 .
\end{aligned}
$$

Thus $\mathfrak{L}_{\gamma}(f)$ is not starlike for all $0<\gamma<1$. For general $\gamma=\rho e^{i \phi}, 0<\rho$ $<1$, consider $f(z)=z+a_{2} z^{2}, a_{2}=\frac{1}{2} e^{i \phi}$. Then the behaviour of $\mathfrak{E}_{\gamma}(f)$ near $e^{i \phi}$ yields a similar result.

If $\mathcal{L}$ is a nontrivial linear homeomorphism of $\mathscr{E S C} K$, then the example $f(z)=[(1+z) /(1-z)]^{1 / 2}-1 \in K$ can be used to show $\mathfrak{L}(K) \oplus S$. Similarly the example $f(z)=z+\frac{1}{2} e^{i \phi} z^{2}$ shows that $\mathfrak{L}(S t) \nsubseteq S$, for $\mathcal{L}$ a nontrivial linear homeomorphism of EFCSt. The techniques and examples just used also show that the only linear homeomorphism from $K$ to $S t$ is $\mathfrak{L}(f(z))=z f^{\prime}(z)$ followed by a rotation. With these theorems in mind the positive result of the next section is surprising.

2. Let $\mathcal{P}$ be the compact convex set in $\mathscr{Q}$ with positive real part and $f(0)=1$. Then

$$
\mathscr{E} \mathscr{P}=\{f: f(z)=(1+x z) /(1-x z),|x|=1\} .
$$

In [9] and in a paper to appear [10], T. Sheil-Small has investigated linear operators on $\mathcal{Q}$ which are maps from $\mathscr{P}$ to $\mathscr{P}$. The following results are restricted to homeomorphisms of $\mathcal{P}$.

THEOREM 6. Let $\mathfrak{L}$ be a continuous linear operator on $\mathbb{Q}$, then the following statements are equivalent:

(i) $\mathcal{L}$ is a one-to-one mapping of $\mathcal{P}$ onto $\mathcal{P}$.

(ii) $\mathcal{L}$ is a one-to-one mapping of $\mathcal{E} \mathcal{P}$ onto $\mathcal{E} \mathcal{P}$.

If (i) or (ii) holds and $f(z)=\sum_{k=0}^{\infty} b_{k} z^{k} \in \mathbb{Q}$ then

$$
\mathfrak{L}(f)=b_{0}\left(\frac{1+\gamma z}{1-\gamma z}\right)+z \frac{\left(|\gamma|^{2}-1\right)}{(1-\gamma z)^{2}} \sum_{k=1}^{\infty} b_{k}\left[\frac{\bar{\gamma}-z}{1-\gamma z}\right]^{k-1}
$$

$$
= \begin{cases}b_{0}\left[\frac{\bar{\gamma}-\gamma z^{2}}{(1-\gamma z)(\bar{\gamma}-z)}\right]+z \frac{\left(|\gamma|^{2}-1\right)}{(1-\gamma z)(\bar{\gamma}-z)} f\left(\frac{\bar{\gamma}-z}{1-\gamma z}\right), & z \neq \bar{\gamma}, \\ b_{0}\left(\frac{1+|\gamma|^{2}}{1-|\gamma|^{2}}\right), & z=\bar{\gamma} .\end{cases}
$$

Proof. The proof follows exactly the line of proof in Theorems 2 and 3 upon noting that $\mathscr{P}$ is convex and compact. 
LEMMA 2. If $f \in S t$ and $\rho$ is given $(0<\rho<1)$, then $f$ can be uniformly approximated in $|z| \leqslant \rho$ by functions of the form

$$
F(z)=z / \prod_{k=1}^{n}\left(1-\varepsilon_{k} z\right)^{\lambda_{k}}
$$

where $\left|\varepsilon_{k}\right|=1, \lambda_{k} \geqslant 0$ and $\sum_{k=1}^{n} \lambda_{k}=2$.

The proof of this lemma is generally known and is a consequence of a similar assertion for functions with positive real part in $\Delta$ as given by the Herglotz formula.

THEOREM 7. Let $\mathfrak{L}$ be a linear operator on $\mathbb{Q}$ which is a linear homeomorphism of $\mathcal{P}$. Then if $f \in S t, \mathcal{L}(f)$ is starlike with respect to the origin but $\mathfrak{L}(K) \nsubseteq K$.

Proof. If $f \in S t$ then $f$ can be approximated by functions $F$ given by (7). Therefore $\mathfrak{L}(f)$ is approximated by $\mathcal{L}(F)$. If we show each $\mathfrak{L}(F)$ is of the form (7), then $\mathcal{L}(f)$ will be starlike. From (6) and (7)

$$
\mathcal{L}(F)(z)=\frac{|\gamma|^{2}-1}{\prod_{k=1}^{n}\left(1-\varepsilon_{k} \bar{\gamma}\right)^{\lambda_{k}}} \cdot \frac{z}{\prod_{k=1}^{n}\left(1-\delta_{k} z\right)^{\lambda_{k}}}
$$

where $\delta_{k}=\left(\gamma-\varepsilon_{k}\right) /\left(1-\varepsilon_{k} \bar{\gamma}\right)$.

Since $\left|\delta_{k}\right|=1$ we see that $\mathscr{L}(F)$ has the desired form (7), thus $\mathcal{L}(F)$ is starlike with respect to the origin, although not in $S t$ as $\mathscr{L}(f)$ is not properly normalized.

The function $f(z)=\frac{1}{2} \log [(1+z) /(1-z)]$ again shows that $\mathscr{L}(K) \nsubseteq K$.

The author would like to acknowledge helpful communications with Dr. T. Sheil-Small during the preparation of this paper.

\section{REFERENCES}

1. L. Brickman, T. H. MacGregor and D. R. Wilken, Convex hulls of some classical families of univalent functions, Trans. Amer. Math. Soc. 156 (1971), 91-107. MR 43 \#494.

2. J. Clunie and F. R. Keogh, On starlike and convex schlicht functions, J. London Math. Soc. 35 (1960), 229-233. MR 22 \# 1682.

3. N. Dunford and J. T. Schwartz, Linear operators. I. General theory, Pure and Appl. Math., vol. 7, Interscience, New York, 1958. MR 22 \#8302.

4. M. G. Haplanov, Infinite matrices in an analytic space, Uspehi Mat. Nauk 11 (1956), no. 5(71), 37-44; English transl., Amer. Math. Soc. Transl. (2) 13 (1960), 177-183. MR 18, 810; 22 \#3977.

5. F. W. Hartmann and T. H. MacGregor, Matrix transformations of univalent power series, J. Austral. Math. Soc. 28 (1974), 419-435.

6. T. H. MacGregor, Hull subordination and extremal problems for starlike and spiral-like mappings, Trans. Amer. Math. Soc. 183 (1973), 499-510. MR 49 \# 3104.

7. R. M. Robinson, Univalent majorants, Trans. Amer. Math. Soc. 61 (1947), 1-35. MR 8 \# 370 .

8. St. Ruscheweyh and T. Sheil-Small, Hadamard products of schlicht functions and the PolyaSchoenberg conjecture, Comment. Math. Helv. 48 (1973), 119-135. MR 48 \#6393. 
9. T. B. Sheil-Small, Some linear operators in function theory, Sympos. on Complex Analysis, Canterbury, 1973.

10. - On the preservation of linear structure for analytic and harmonic functions (to appear).

11. A. Wilansky, Functional analysis, Blaisdell, New York, 1964. MR 30 \#425.

Department of Mathematics, Villanova University, Villanova, Pennsylvania 19085 